\title{
Mitroflow Aortic Bioprosthesis Failure in Type B Aortic Dissection: Preventive Left Main Stenting in Transapical Transcatheter Aortic Valve Implantation Strategy
}

\author{
Giovanni Alfonso Chiariello, b, c, Emmanuel Villa ${ }^{\mathrm{b}}$, Antonio Messina ${ }^{\mathrm{b}}$, Stefano Marwan Manginia, b, \\ Massimiliano Rozzi ${ }^{b}$, Margherita Dalla Tomba ${ }^{\mathrm{b}}$, Ornella Leonzi ${ }^{\mathrm{b}}$, \\ Giovanni Troise ${ }^{\mathrm{b}}$
}

\begin{abstract}
Mitroflow aortic prosthesis dysfunction in case of complex vascular disease is considered a challenging scenario. Because of the high risk for surgical reoperation and the presence of chronic aortic dissection originated from a calcified Kommerel diverticulum, we considered to perform a transapical valve-in-valve transcatheter aortic valve implantation (TAVI) procedure. Myocardial ischemia is a dreadful complication reported in valve-in-valve TAVI procedures, mainly in patients with degenerated Mitroflow aortic bioprostheses. Because of the narrow shape of Valsalva sinuses and the short distance between Mitroflow annulus and left coronary ostium, to overcome the risk of possible Mitroflow leaflets displacement during TAVI expansion thus overlapping coronary ostia, we performed a preventive angioplasty. Then, we implanted a bare metal stent on the left main protruding in the aortic root. At 3 years follow-up the patient was in good clinical conditions.
\end{abstract}

Keywords: Aortic valve; Aortic dissection; Valve-in-valve; TAVI; Coronary stenting

\section{Introduction}

Valve-in-valve transcatheter aortic valve implantation (ViV TAVI) for degenerated aortic bioprostheses has been reported as an effective procedure in high risk patients. The transfemoral approach is the route of choice, but the transapical or transaortic approaches could be considered in case of severe peripheral vascular disease. Despite the good results obtained, a relevant risk of coronary ostia obstruction and myocardial

Manuscript submitted December 23, 2017, accepted January 8, 2018

${ }^{a}$ Cardiovascular Sciences Department, Catholic University of The Sacred Heart, Rome, Italy

${ }^{\mathrm{b}}$ Cardiac Surgery, Poliambulanza Foundation, Brescia, Italy

${ }^{\mathrm{c} C}$ Corresponding Author: Giovanni Alfonso Chiariello, Cardiovascular Sciences Department, Catholic University of The Sacred Heart, Largo Agostino Gemelli 8,00168 Rome, Italy. Email: gio.chiariello88@tiscali.it

doi: https://doi.org/10.14740/cr666w ischemia has been observed during ViV TAVI procedures.

\section{Case Report}

An 80-year-old woman was admitted at emergency department for pulmonary edema. She was previously diagnosed with chronic type B aortic dissection arising from a Kommerell diverticulum and in 2008 she underwent aortic valve replacement (Mitroflow $n^{\circ} 23$, Sorin, Saluggia, Italy) without correction of the aortic dissection (stable diameters, visceral perfusion from both lumens). Five years later, central severe aortic regurgitation appeared with moderate to severe mitral regurgitation, with reduced ejection fraction $(\mathrm{EF})$ and pulmonary hypertension. In that occasion, chest X-rays was remarkable for the calcification of the vascular knobs (Fig. 1 A-D).

The case was discussed by our internal Heart-Team. The estimated operative risk of the conventional redo aortic valve replacement was $32.5 \%$ (EuroSCORE II). A transcatheter strategy (TAVI) was evaluated but the transfemoral route and other transarterial ways were discarded due to aortic dissection. The transapical route was then chosen and a valve-in-valve TAVI performed (SAPIEN XT n ${ }^{\circ} 23$, Edwards Lifesciences, Irvine, CA).

We were conscious of risk of myocardial ischemia after a valve-in-Mitroflow procedure due to the peculiar design of this prosthesis (pericardial mounted outside the stent to optimize flow) and the unfavorable aortic root anatomy (narrow Valsalva sinuses and short distance between Mitroflow annulus and left coronary ostium). This structure may cause possible dislocation of Mitroflow pericardial leaflets overlapping coronary ostia during balloon expansion or TAVI valve deployment: so TAVI was done after protection of the left coronary artery with two intracoronary wires. Although without electrocardiographic alterations, considering the encumbrance of the Mitroflow leaflets, a protruding $5 \times 20 \mathrm{~mm}$ bare metal stent was implanted in the left main (Fig. 1E). Postoperative echocardiogram showed the correct positioning of the TAVI bioprosthesis with acceptable transprosthetic gradients and good EF (peak gradient $30 \mathrm{~mm}$ $\mathrm{Hg}$, mean gradient $19 \mathrm{~mm} \mathrm{Hg}$, EF 53\%). Postoperative course was uneventful. At 3 years follow-up, patient was in good clinical conditions. Echocardiogram showed the absence of aortic bioprosthesis failure (peak gradient $36 \mathrm{~mm} \mathrm{Hg}$, mean $21 \mathrm{~mm}$ 


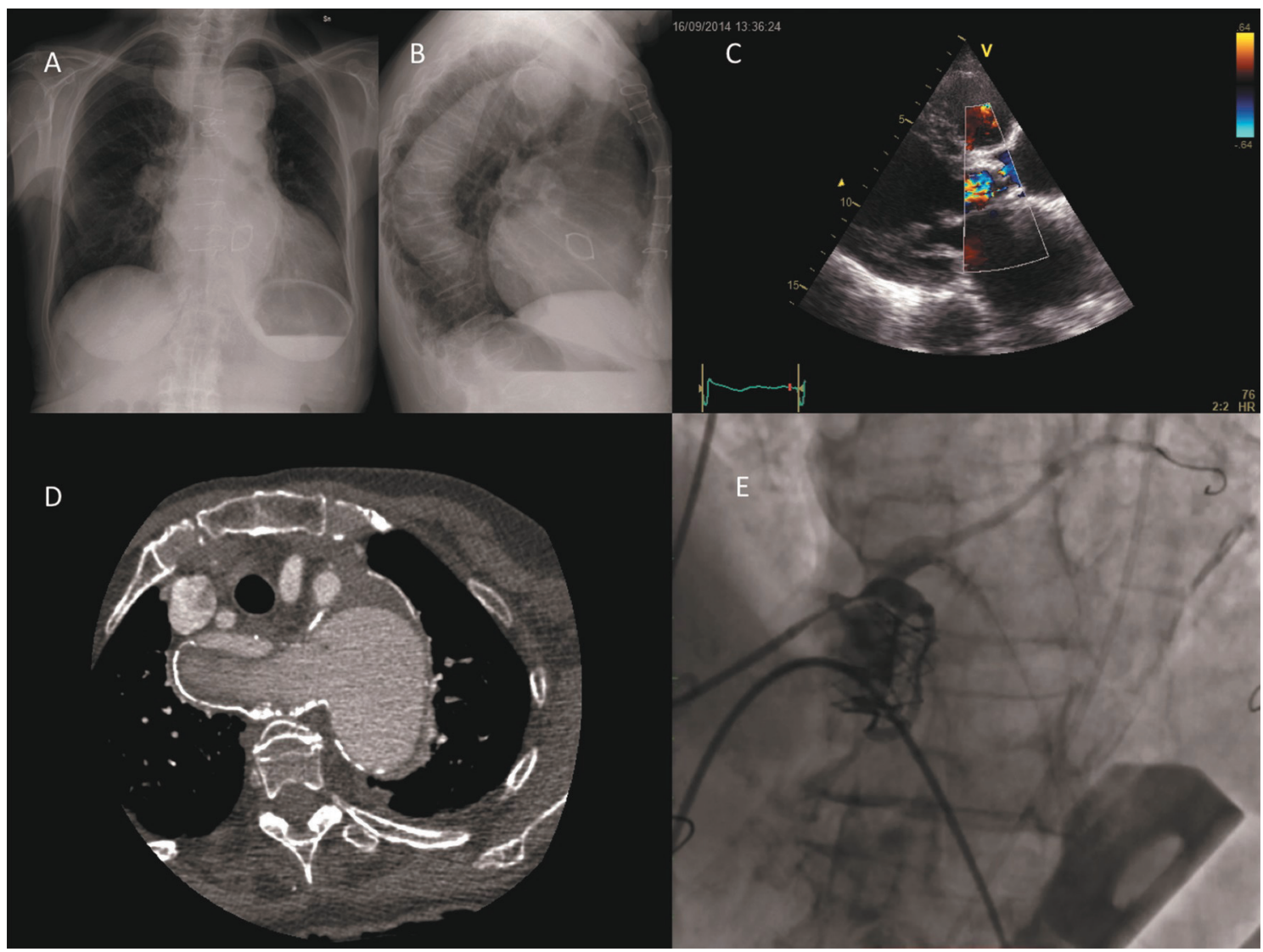

Figure 1. (A-B): Chest X-ray in posteroanterior and laterolateral view. Calcifications of the vascular knobs (aortic arch on the left, right subclavian artery from the diverticulum on the right) and of the descending aorta are evident. (C): Severe aortic regurgitation from Mitroflow prosthesis dysfunction. (D): CT scan shows chronic type B aortic dissection and the calcified Kommerell diverticulum. (E): Intraprocedural angiography shows stenting of the left main after transapical Sapien XT released.

$\mathrm{Hg}$ ) and absence of left ventricular dysfunction (mean gradient $16 \mathrm{~mm} \mathrm{Hg}, \mathrm{EF} \mathrm{55 \% )}$ ) and mild to moderate mitral regurgitation. The bare metal stent previously implanted in the left main protruding in the aortic root was normally positioned (Fig. 2).

\section{Discussion}

Several authors reported a worrisome early degeneration of Mitroflow bioprosthesis, mainly for small sizes, likely for the absence of an effective antimineralization treatment in first models such as Mitroflow LXA $[1,2]$. ViV TAVI has been reported as an effective, fast, and less invasive treatment to overcome risks of redo operation mainly when surgical reoperation with a sutured or a sutureless prosthesis is contraindicated. However, ViV TAVI in small Mitroflow prostheses (19 - 21 $\mathrm{mm}$ ) is associated with a risk of high postoperative gradients and risk of coronary ostia obstruction and acute myocardial ischemia [3-5]. In this case, of a degenerated Mitroflow $23 \mathrm{~mm}$, ViV TAVI was considered feasible, and we didn't observe high postoperative gradients and patients-prosthesis mismatch. Coronary obstruction is a disastrous complication associated with high mortality. The global valve-in-valve registry reported an overall coronary obstruction rate of $3.5 \%$ and nearly $8 \%$ in degenerated Mitroflow bioprosthesis (all sizes included) [3-6].

High-risk features for intraprocedural myocardial ischemia include low-lying coronary ostia with a short distance between the annulus of the degenerated prosthesis and coro-

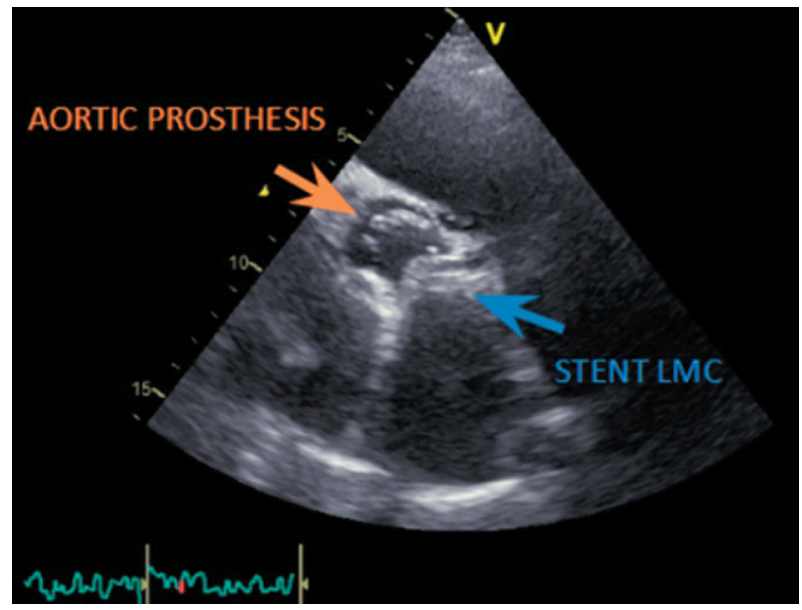

Figure 2. Late follow-up echocardiogram showing the correct positioning both of the TAVI prosthesis and of the stent implanted in the left main. 
nary ostia, short and narrow Valsalva sinuses, supra-annular surgical implantation of the first bioprosthesis, and the lowprofile valve design with pericardial leaflets mounted outside an internal stent frame [4-6].

To prevent a periprocedural myocardial ischemia due to prosthesis leaflets stucked up against the coronary ostia, wiring of the left main was carried on for a faster potential revascularization in case myocardial ischemia during the procedure. In these conditions, a coronary angioplasty may be risky and possibly hampered by the struts of the new valve and the pericardial leaflets of the old Mitroflow prosthesis.

Indeed, preventive left main stenting was also performed in the same session before TAVI valve deployment, as an additional protection of the narrow access to the left main.

\section{Conclusions}

In cases of impracticable peripheral accesses such as for diffuse severe atherosclerotic disease or aortic dissection as in this case, transapical access for ViV TAVI with coronary protection could provide an effective way to the surgeon to replace a degenerated Mitroflow-type aortic valve in non-operable patients. Preventive stenting of the left main with a coronary stent protruding in the aortic root may confer a greater safety to the procedure.

\section{Conflict of Interest}

No author has any conflict of interest to disclose.

\section{Financial Support}

This research received no specific grant from any funding agency, commercial or not-for-profit sectors.

\section{Author Contributions}

All authors have contributed and approved the final version of this manuscript.

\section{References}

1. De Paulis R, D'Aleo S, Bellisario A, Salica A, Weltert LP, Scaffa R, Wolf LG, et al. The fate of small-size pericardial heart valve prostheses in an older patient population. J Thorac Cardiovasc Surg. 2017;153(1):31-39 e32.De Paulis R, D'Aleo S, Bellisario A, Salica A, Weltert LP, Scaffa $\mathrm{R}$, Wolf LG, et al. The fate of small-size pericardial heart valve prostheses in an older patient population. J Thorac Cardiovasc Surg2017;153(1):31-39e32

2. Bassano C, Gislao V, Bovio E, Melino S, Tropea I, Saitto G, Pugliese M, et al. An unexpected risk factor for early structural deterioration of biological aortic valve prostheses. Ann Thorac Surg. 2017.

3. Camboni D, Holzamer A, Florchinger B, Debl K, Endeman D, Zausig Y, Maier LS, et al. Single institution experience with transcatheter valve-in-valve implantation emphasizing strategies for coronary protection. Ann Thorac Surg. 2015;99(5):1532-1538.

4. Webb JG, Dvir D. Transcatheter aortic valve replacement for bioprosthetic aortic valve failure: the valve-in-valve procedure. Circulation. 2013;127(25):2542-2550.

5. Gurvitch R, Cheung A, Ye J, Wood DA, Willson AB, Toggweiler S, Binder R, et al. Transcatheter valve-in-valve implantation for failed surgical bioprosthetic valves. J Am Coll Cardiol. 2011;58(21):2196-2209.

6. Dvir D, Webb J, Brecker S, Bleiziffer S, Hildick-Smith D, Colombo A, Descoutures F, et al. Transcatheter aortic valve replacement for degenerative bioprosthetic surgical valves: results from the global valve-in-valve registry. Circulation. 2012;126(19):2335-2344. 